\title{
Evaluación del Estiércol de Vaca como Inóculo en la Digestión Anaerobia Termófila de Residuos Sólidos Urbanos
}

\author{
Ricardo Camacho, Héctor S. Villada y José L. Hoyos \\ Universidad del Cauca, Facultad de Ciencias Agrarias, Grupo de investigación CYTBIA y ASUBAROIN, \\ Vereda Las Guacas, Popayán, Colombia. (e-mail: rcmunoz@unicauca.edu.co; villada@unicauca.edu.co)
}

Recibido Oct. 21, 2016; Aceptado Dic. 23, 2016; Versión final Feb. 2, 2017, Publicado Jun. 2017

\begin{abstract}
Resumen
El presente trabajo evaluó la utilización de estiércol de vaca como inóculo durante el arranque de un sistema de digestión anaerobia termófila seca (DATS) de la fracción orgánica de residuos sólidos urbanos (FORSU). Se sabe que el arranque es la etapa de mayor complejidad durante la digestión anaerobia y exige la elección de un inoculo apropiado y disponible. La estrategia se dividió en tres etapas: (1) aclimatación del inóculo, (2) adición del residuo y (3) re-inoculación. Los porcentajes de metano revelaron una elevada actividad metanogénica y rápida aclimatación del inóculo al rango termófilo. Durante la etapa de incorporación de la FORSU los niveles AGV, AGV/Alc y alcalinidad tienden a incrementar. El pH desciende y requiere ajuste continuo. Tras la re-inoculación, el pH alcanza un valor por encima de 7.47, pero tiende igualmente a descender, mientras que AGV y AGV/Alc se mantienen sobre los $12,58 \mathrm{~g} / \mathrm{ml}$ y 0,83 respectivamente. Los parámetros medidos sugieren que la mezcla sustrato/inoculo sufrió una acumulación excesiva de AGV lo que indica que el residuo requiere niveles mayores de inoculación.
\end{abstract}

Palabras clave: residuos sólidos; digestión anaerobia; termófilo; estiércol de vaca; arranque

\section{Evaluation of Cow Manure as Inoculum in the Thermophilic Anaerobic Digestion of Municipal Solid Waste}

\begin{abstract}
In this study, cow manure was evaluated as inoculum in the star-up of the dry thermophilic anaerobic digestion process (DTAD) of municipal solid waste (MSW). Start-up is the more complex stage in anaerobic digestion in which an appropriate and available inoculum is required. The start-up was separated in three stages: (1) inoculum acclimation, (2) waste incorporation and (3) re-inoculation. Methane percentages revealed a high methanogenic activity and rapid inoculum acclimation to the thermophilic range. During the stage 2 high VFA and VFA/Alk was observed. The $\mathrm{pH}$ decreases and adjustment is continuously required. In the stage 3, the pH reaches a value higher than 7.47 but it tends to decrease rapidly. On the other hand VFA and VFA/Alk reach $12,58 \mathrm{~g} / \mathrm{ml}$ y 0,83 respectively. The evaluated parameters suggest that the substrate/inoculum mixture suffered an excessive VFA accumulation that shows that the waste required higher inoculation levels.
\end{abstract}

Keywords: solid waste; anaerobic digestion; thermophilic; cow manure; start-up 


\section{INTRODUCCIÓN}

La abundante generación de residuos sólidos urbanos, que se estima en cerca 1,3 billones de toneladas anuales, se ha convertido en un serio problema social y ambiental a nivel mundial (Ouda y Raza, 2014). La fracción orgánica de residuos solios urbanos (FORSU) puede ser tratada de manera eficiente mediante digestión anaerobia, tecnología que ha adquirido gran importancia especialmente en países europeos dadas las ventajas que presenta respecto a los tratamientos aerobios (Blanco, 2010). La digestión anaerobia termófila seca DATS es un proceso complejo en el cual la materia orgánica es degradada paulatinamente en ausencia de oxígeno hasta un gas (biogás) conformado principalmente de dióxido de carbono y metano. Esta alternativa aplicada al tratamiento de la FORSU, permite la reducción de microorganismos patógenos, del volumen del residuo y de la materia orgánica, además, provee un recurso energético valioso (biogás) y un efluente (digestato) con capacidad de ser empleado en la enmienda orgánica de suelos. La DATS ofrece también beneficios ambientales como la reducción de olores o la disminución de los gases de efecto invernadero (De Francisci et al., 2015).

La digestión anaerobia termófila exhibe ciertas ventajas frente a sus contrapartes ya que permite un incremento en las cargas orgánicas y disminución de los tiempos de retención, también aumenta la velocidad de arranque del sistema, evita la formación de espumas e incrementa la eficacia de la destrucción de patógenos. Por otra parte, la DATS de la FORSU tiene como principales ventajas, la reducción del espacio requerido en el reactor, menor utilización de agua para disolución, mayores índices de generación de gas, y facilidades en la disposición del digestato; sin embargo, debido a su elevada biodegradabilidad, la FORSU puede generar problemas de inestabilidad cuando es sometida a DAT en altas concentraciones de solidos (Khalid et al., 2011).

El arranque (establecimiento de comunidades microbianas) de la DATS es la etapa más crítica del proceso y depende entre otras del tipo de inóculo y la relación inóculo/sustrato (Motte et al., 2013). Los inóculos empleados provienen generalmente de sistemas de degradación operantes y preferiblemente mantenidos a las condiciones de trabajo propuestas para el nuevo montaje. Debido a la alta distribución de la digestión anaerobia en Europa (De Baere y Mattheeuws, 2013) estos procesos son más agiles que en países en vía de desarrollo en los que no siempre es factible efectuar la escogencia del inóculo debido a su escasa disponibilidad. No obstante, se puede emplear fuentes no aclimatadas como el estiércol de vaca para el arranque de sistemas anaerobios (El-Fadel et al., 2013) El objetivo del presente trabajo fue estudiar el uso el estiércol de vaca obtenido directamente del rumen del animal, como inóculo en el arranque para la digestión anaerobia termófila seca de la fracción orgánica de residuos solios urbanos. La estrategia de arranque se dividió en tres etapas: aclimatación del inóculo, adición de la FORSU y re-inoculación, durante las cuales se efectuó seguimiento de los principales parámetros operativos: volumen de biogás ( $\mathrm{V}_{\text {gas }}$ ), alcalinidad total (Alc), ácidos grasos volátiles totales (AGV), porcentajes de metano en gas $\left(\% \mathrm{CH}_{4}\right)$ y pH (Jain et al., 2015).

\section{MATERIALES Y MÉTODOS}

Se describe el reactor anaerobio, el inóculo y el sustrato, para luego presentar el procedimiento experimental y los métodos analíticos usados.

\section{Reactor Anaerobio}

Se utilizó un reactor de $20 \mathrm{~L}$ en acero inoxidable, mantenido a temperatura constante mediante reflujo de agua. El mezclado se efectuó con la ayuda de tres juegos de paletas dobles dispuestas de manera equidistante a lo largo del reactor e inclinadas $30^{\circ}$ respecto al eje de agitación. El biogás fue recolectado en una bolsa TEDLAR de $40 \mathrm{~L}$ para su posterior cuantificación y análisis.

\section{Inóculo}

Se empleó estiércol de vaca obtenido directamente del rumen del animal, suministrado por el centro de sacrificio de la ciudad de Popayán - Colombia (Forster-Carneiro et al., 2007). El estiércol fue almacenado en recipientes plásticos herméticos desinfectados con hipoclorito de sodio (200 ppm), almacenado a temperatura ambiente hasta su utilización y caracterizado mediante la valoración de Solidos totales (ST), solidos volátiles (SV), Alc y AGV.

\section{Sustrato}

Para la preparación del sustrato se seleccionó la FORSU proveniente de diferentes plazas de mercado de la ciudad de Popayán. La FORSU se secó a $50^{\circ} \mathrm{C}$ (Forster-Carneiro et al., 2008) para facilitar su manipulación y reducción de tamaño. El material seco fue triturado, tamizado (malla de $5 \mathrm{~mm}$ ), mezclado y almacenado hasta su utilización (Forster-Carneiro et al., 2008). La caracterización fisicoquímica de la FORSU fue efectuada por el laboratorio de externo y es presentada en la Tabla 1. 


\section{Procedimiento experimental}

La estrategia empleada para el arranque de la DATS de FORSU consistió de tres etapas: En la Etapa 1 (aclimatación del inóculo), se pesaron $8,7 \mathrm{~kg}$ de estiércol de vaca a los que se le incorporó $1,6 \mathrm{~kg}$ de agua libre de cloro (Forster-Carneiro, 2005). La mezcla fue incorporada al reactor anaerobio y se mantuvo durante 30 días a temperatura de operación $\left(55^{\circ} \mathrm{C}\right)$ con agitación a $15 \mathrm{rpm}$ en ciclos de 2 horas (encendido y apagado cada 2 horas) (Fernández, 2008). En la etapa 2 (incorporación de la FORSU), se adicionaron 4,2 kg del sustrato (humedad de 3,6\%) y se mantuvieron las condiciones de operación previas (temperatura y agitación) (Forster-Carneiro 2005). En la etapa 3 (re-inoculación) se empleó estiércol de vaca sin aclimatación ni dilución. El seguimiento de los parámetros se llevó a cabo a lo largo de todo el experimento e incluyó medición de pH, AGV, Alc, SV, ST, $\mathrm{V}_{\text {gas }}$ y \% $\mathrm{CH}_{4}$. La corrección de $\mathrm{pH}$ fue realizada mediante la adición de $\mathrm{NaOH} 6 \mathrm{~N}$ y una mezcla 2:1 de $\mathrm{NaHCO}_{3}: \mathrm{CaCO}_{3}$ en momentos donde el parámetro se ubicó por debajo de 6.8

\section{Métodos analíticos}

Se empleó protocolos del Standard Methods (SM) (American Public Health Association et al., 1981). La muestra obtenida directamente del reactor se valoró en términos de SV y ST (SM 2540), posteriormente se realizó una mezcla de $10 \mathrm{~g}$ de muestra en $100 \mathrm{ml}$ de agua destilada y se agitó durante 2 horas hasta su completa lixiviación. La muestra lixiviada se utilizó para la medición de pH (SM 4500B) y Alc (SM 2320B) por pontenciometría empleando un pH-metro SI ANALYTICS Lab 860 calibrado en dos puntos con buffer Fluka BS. La muestra lixiviada fue a su vez filtrada secuencialmente a través de un filtro de fibra de vidrio de 0,45 $\mu \mathrm{m}$ y el filtrado se usó en la valoración de AGV (SM 5560B). El análisis de composición del biogás se realizó mediante un sensor de metano por infrarrojo no dispersivo (NDIR) marca HITECH HITOX IR 600 y el volumen de biogás generado mediante desplazamiento de una columna de agua en probeta invertida de 2 L. Los volúmenes medidos fueron transportados a volumen en condiciones estándares de presión y temperatura por litro de espacio ocupado en el reactor (Yagi et al., 2012).

\section{RESULTADOS Y DISCUSIÓN}

El presente trabajo estudio estiércol de vaca como inoculo en el arranque de la digestión anaerobia termófila $\left(55^{\circ} \mathrm{C}\right)$ seca $(20 \% \mathrm{ST})$ de FORSU. Inicialmente se aclimato el estiércol de vaca a rango termófilo y posteriormente se incorporó FORSU al sistema. En la Tabla 1 se presenta los principales parámetros de caracterización tanto del inóculo como de la FORSU empleada. Las figuras 1 a 3 proporcionan los resultados de la evolución de $\mathrm{pH}$, Alc, $\mathrm{AGV}$, relación AGV/Alc, $\mathrm{V}_{\text {gas }}$ y $\% \mathrm{CH}_{4}$ de la mezcla de inóculo - FORSU durante 100 días de digestión

\section{Características del inóculo y la FORSU}

Tabla 1. Caracterización del inoculo y FORSU empleado en el presente estudio. Parámetro * Valores en base húmeda ${ }^{* *}$ Valores en base seca ${ }^{* *}$ : Laboratorio de bromatología y abonos orgánicos de la Universidad de Nariño.

\begin{tabular}{|c|c|}
\hline & Valor \\
\hline \multicolumn{2}{|c|}{$F_{F O R S U^{* * *}}$} \\
\hline Humedad $(\mathrm{g} / 100 \mathrm{~g})^{*}$ & 3,7 \\
\hline Solidos Totales $(\mathrm{g} / 100 \mathrm{~g})^{*}$ & 96,3 \\
\hline Solidos Volátiles $(\mathrm{g} / 100 \mathrm{~g})^{\star \star}$ & 89,7 \\
\hline Ceniza $(\mathrm{g} / 100 \mathrm{~g})^{\star \star}$ & 10,3 \\
\hline Carbono Orgánico Total $(\mathrm{g} / 100 \mathrm{~g})^{\star \star}$ & 52,8 \\
\hline Nitrógeno $(\mathrm{g} / 100 \mathrm{~g})^{\star *}$ & 1,79 \\
\hline Calcio $(\mathrm{g} / 100 \mathrm{~g})^{\star \star}$ & 0,56 \\
\hline Fosforo $(\mathrm{g} / 100 \mathrm{~g})^{\star \star}$ & 0,21 \\
\hline Magnesio $(\mathrm{g} / 100 \mathrm{~g})^{\star \star}$ & 0,26 \\
\hline Potasio $(\mathrm{g} / 100 \mathrm{~g})^{\star \star}$ & 3,03 \\
\hline Azufre $(\mathrm{g} / 100 \mathrm{~g})^{\star *}$ & 0,18 \\
\hline Hierro $(\mathrm{mg} / \mathrm{Kg})^{\star \star}$ & 10,17 \\
\hline Manganeso $(\mathrm{mg} / \mathrm{Kg})^{* *}$ & 96 \\
\hline Zinc $(\mathrm{mg} / \mathrm{Kg})^{* *}$ & 35,6 \\
\hline Cobre $(\mathrm{mg} / \mathrm{Kg})^{\star \star}$ & 5,72 \\
\hline \multicolumn{2}{|c|}{ Inóculo } \\
\hline Solidos Totales (\%)* & 15,3 \\
\hline Solidos Volátiles $(\%)^{\star *}$ & 79,3 \\
\hline Alcalinidad $\left(\mathrm{gCaCO}_{3} / \mathrm{L}\right)^{*}$ & 4,3 \\
\hline Ácidos grasos volátiles $\left(\mathrm{g} \mathrm{AcH}^{+} / \mathrm{L}\right)^{*}$ & 1,3 \\
\hline
\end{tabular}


Evolución de los parámetros de seguimiento

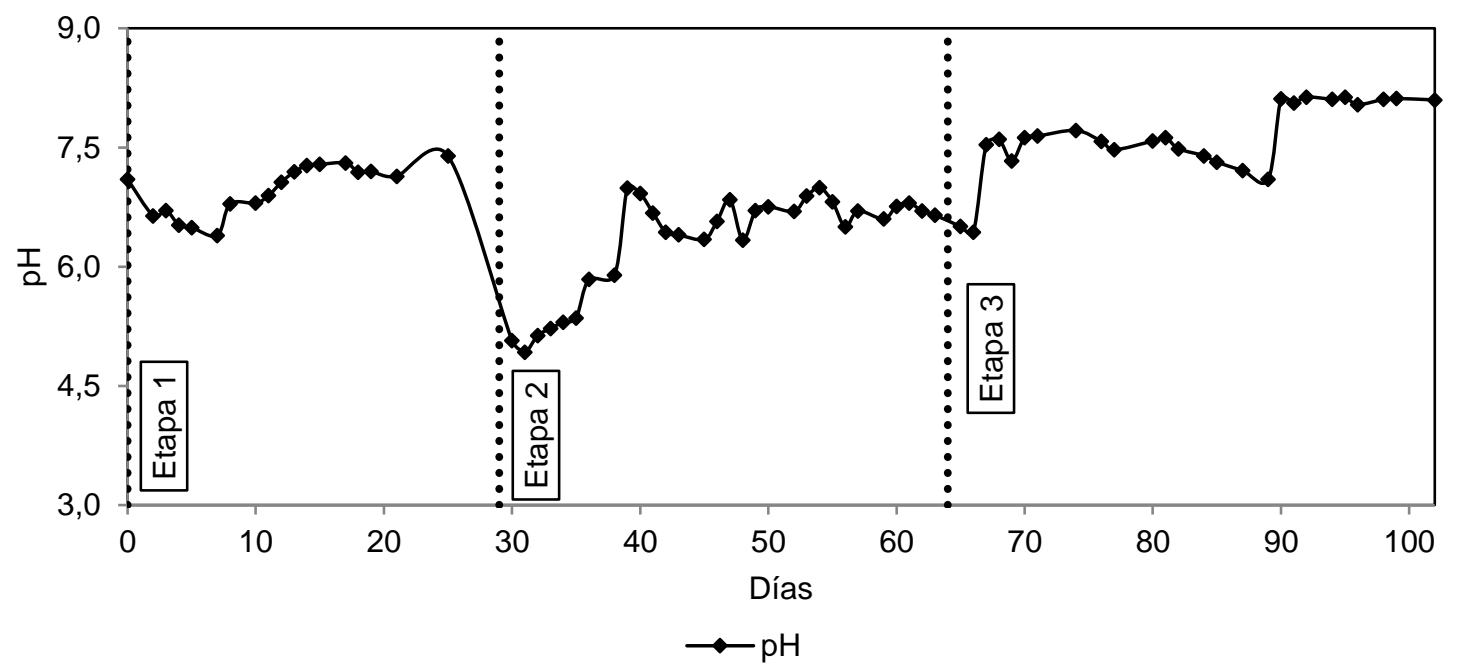

Fig. 1: Evolución del pH de la mezcla en digestión anaerobia

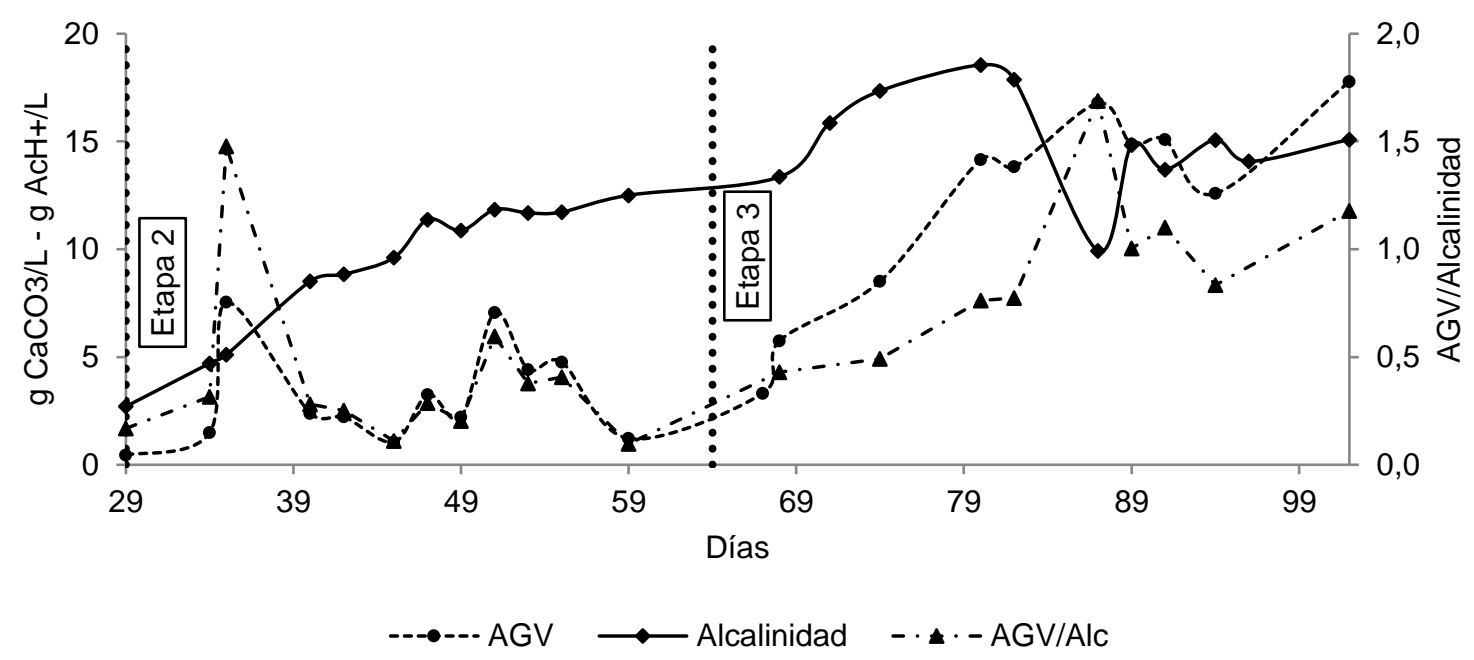

Fig. 2: Evolución de Alc, AGV y relación AGV/Alc de la mezcla en digestión anaerobia

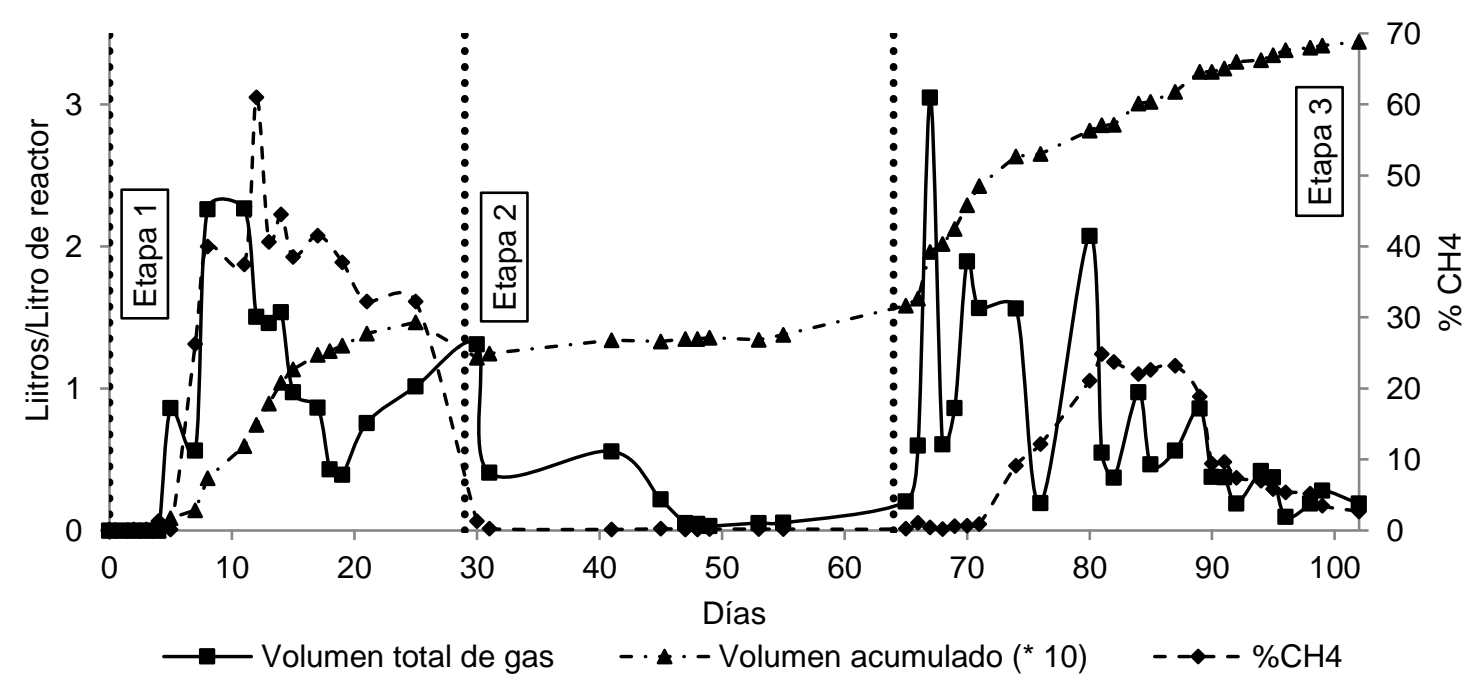

Fig. 3: Evolución del $\mathrm{V}_{\text {gas }} \mathrm{y} \% \mathrm{CH}_{4}$ generado por la mezcla en digestión anaerobia. 


\section{Etapa 1. Aclimatación del inóculo}

Durante los primeros 5 días de DATS, el pH del medio descendió, posteriormente incrementó (día 8 a 11) y se estabilizó al término día 11 (Fig. 1). Lo anterior estaría relacionado con una disminución de AGV producto de la actividad sinérgica de arqueas metanogénicas hidrogenótrofas y acetoclásticas (Rowse, 2011) y el establecimiento de un tampón debido a la degradación de proteínas y la disolución de dióxido de carbono (Challen-Urbanic et al., 2011). Por otro lado, el incremento del \% $\mathrm{CH}_{4}$ (Fig. 3) desde el día 5 (0,15 \%) al 12 $(60,55 \%)$, reveló una elevada actividad de las arqueas metanogénicas acetoclásticas que coincide con el comportamiento del $\mathrm{pH}$ descrito anteriormente. Sin embargo, el \% $\mathrm{CH}_{4}$ disminuyó desde el día 12 al 18 debido al agotamiento de los sustratos metanogénicos. Valencia et al., (2011), quienes estudian la DA de fluidos ruminales de vaca con $\mathrm{ST}=6 \%$, describen una evolución de $\mathrm{pH}$ en rango mesófilo $\left(35{ }^{\circ} \mathrm{C}\right)$ similar a la observada en el presente trabajo, sin embargo \% $\mathrm{CH}_{4}$ para su caso alcanza cerca del $90 \%$. Canepa y Oliver (2013) por su parte evaluaron la DA de rumen disuelto en un biodigestor tipo cúpula y obtuvieron niveles de $\% \mathrm{CH}_{4}$ cercanos a los máximos observados en este ensayo.

La producción de gas (Fig. 3) reveló una fase de latencia durante los 4 primeros días de la etapa 1 propia de un cambio en el régimen de temperatura (Gerardi, 2003). No obstante el $V_{\text {gas }}$ incrementó los siguientes 7 días (hasta un máximo de 2,27 Litros/Litros de reactor $\left(L_{/} / L_{R}\right)$ ) debido a la hidrólisis, acidogénesis y acetogénesis de las sustancias solubilizadas (Mao et al., 2015). A continuación el $\mathrm{V}_{\text {gas }}$ desciendió paulatinamente hasta el día 18 probablemente por el agotamiento de estas sustancias.

\section{Etapa 2. Adición de la FORSU al sistema}

Como se observa en la Fig. 1, tras la adición de la FORSU (día 29), el pH sufrió un descenso inicial a 5,07 debido a las características ácidas del residuo. Así mismo, el alto contenido de materia orgánica, su elevada biodegradabilidad (SV/ST = 0.89) (Pavan et al., 2000) y las mayores velocidades específicas de crecimiento de las bacterias acidogénicas respecto a las metanogénicas (Fernández, 2008) pudieron haber generado una elevada producción y acumulación de AGV $\left(0,46\right.$ a 7,0 $\left.\mathrm{gAcH} \mathrm{H}^{+} / \mathrm{L}\right)$ que desembocó igualmente en el descenso del pH (a 4,93) en el día 30. A medida que avanza la DA y como resultado del incremento de la alcalinidad en el medio (Fig. 2), el pH adquirió un comportamiento más estable con descensos diarios menos acusados. Sin embargo, la adición de $\mathrm{NaOH} 6 \mathrm{~N}$ fue siempre necesaria durante este periodo (180 g en promedio para los primeros 11 días y $40 \mathrm{~g}$ para los siguientes 26) ya que decreció constantemente bajo los niveles recomendables $(<6,8)$ (Boontian, 2013). Forster-Carneiro et al. (2007) evidenciaron un comportamiento similar en el arranque de la DA de FORSU en donde AGV incremento de forma considerable hasta alcanzar un valor máximo de $4 \mathrm{~g} / \mathrm{L}$. Esto podría indicar igualmente que los niveles de inoculación en el presente trabajo fueron muy bajos o que el sustrato empleado exhibe una alta susceptibilidad a la degradación biológica. Por otro lado, El-Fadel et al. (2013) señala que el estiércol por si solo puede no llegar a tener suficiente diversidad de microbiota anaerobia para asumir la acidez medio. En ese sentido, Valencia et al. (2011) observaron que una concentración de ST del 9\% durante la DA de rumen bovino, desencadenó una rápida acidificación del sistema debido a que los conglomerados metanogénicos son incapaces de consumir los AGV producidos en las etapas previas de la digestión.

La producción de AGV (Fig. 2) hasta el día 64 tuvo una tendencia creciente y sinuosa, no obstante, la elevada alcalinidad del medio, que durante esta etapa incrementó (comportamiento típico de la fase hidrolítica) de manera continua, pudo contribuir a una incipiente estabilización del pH. Igualmente, como muestra la Fig. 2 la relación AGV/Alc aumento superando los valores recomendables de 0,1 a 0,2 (Khanal, 2011), 0,3 a 0,4 (Brown y Li, 2013) o incluso de 0,8 (Zhu y Jha, 2013).

Durante la etapa 2 no se percibió producción de biogás y los índices de metano descendieron a $0 \%$ (Fig. 3). Este fenómeno fue originado posiblemente debido a la acumulación de AGV que se ubicaron en general sobre los límites de toxicidad recomendables $(0,5$ a $1,5 \mathrm{~g} \mathrm{AcH}+/ \mathrm{L})$ y que son nocivos principalmente para las bacterias metanogénicas (Ariunbaatar et al., 2014). El comportamiento anterior es habitual en materiales altamente biodegradables en la DATS y puede sugerir que los niveles de inoculación (relación sustrato/inóculo) y/o la diversidad o concentración microbiana en el inóculo es insuficiente (Motte et al., 2013).

\section{Etapa 3. Re-inoculación}

En el día 64 de ensayo, de acuerdo con el comportamiento de los AGV (sobre los límites recomendados), Alc, $\mathrm{pH}$ ( $\mathrm{pH}$ no logró estabilizarse naturalmente), $\mathrm{V}_{\text {gas }}$ (producción insignificante) y $\% \mathrm{CH}_{4}$ (no evidenció metano en gas) (Fig. 1, Fig. 2 y Fig. 3) observados durante la etapa 2, puede concluirse que el reactor entró en un estado de acidificación. Se decidió re-inocular sistema mezclando en partes iguales el residuo digerido y estiércol de vaca buscando diluir la concentración de AGV y proveer al medio de alcalinidad y de arqueas metanogénicas capaces de disminuir la concentración de ácido acético (Fernández, 2008). Tras la re-inoculación el pH 
descendió al día 65 (a 6,42) lo que se corrigió con la adición de $\mathrm{NaOH} 6 \mathrm{~N}$ (hasta pH 6,9). Posteriormente los valores se mantuvieron constantes hasta el día 80 en un rango elevado (entre 7,53 a 7,6). Este aumento de $\mathrm{pH}$ pudo haber sido originado debido a la significativa alcalinidad que aportan los estiércoles de vaca (Capela et al., 2008). Igualmente la incorporación de conglomerados metanogénicos pudo haber contribuido al consumo de ácido acético con el consecuente aumento de \% $\mathrm{CH}_{4}$ en el día $79 \%$ (Fig. 3). El \% $\mathrm{CH}_{4}$ en la etapa 3 alcanzó un tope de $24,85 \%$ al día 81 seguido de una disminución leve que concordó también con el inicio de la disminución del $\mathrm{pH}$.

Por su parte, los AGV exhibieron un incremento abrupto y llegaron a alcanzar los $16 \mathrm{~g} \mathrm{AcH} / \mathrm{L}$. A pesar que la alcalinidad también incrementó en esta etapa, al día 82 inició una disminución (de 17,88 gCaCO $/ \mathrm{L}$ a 9,6 $\mathrm{gCaCO}_{3} / \mathrm{L}$ ) vinculada con el consumo de alcalinidad parcial (AP) o bicarbonacea. Este consumo de AP impidió la caída del pH, pero indicó un inicio de la pérdida de la capacidad tampón del sistema. Córdoba et al., 2016) evaluaron la influencia de diferentes inóculos (entre ellos, fluidos ruminales de vaca) en la DA de aguas residuales porcícolas. La alcalinidad en su ensayo incremento también de manera considerablemente durante los primeros 20 días de digestión. Este aumento fue atribuido a la alcalinidad intermedia o acética (debida a AGV) y puede sugerir que en este experimento se produjo igualmente acumulación de acidez. Tanto la elevada producción de AGV (Fig. 2) como la de biogás (3,05 litros de biogás /L reactor (L/LR) en el día 67\% (Fig. 3) en la etapa de re-inoculación pudieron desencadenarse debido a la carga orgánica contenida en el inóculo propiamente y/o debido al suministro de conglomerados microbianos no metanogénicos principalmente acidogénicos y acetogénicos. La caída del pH, Alc al día 80 y el incremento de los AGV puede estar relacionado también con la acumulación excesiva de amonio que a pH mayor a 7 ejerce un efecto ostensiblemente tóxico relacionado con la disociación de $\mathrm{NH}_{4}+$ hacia su forma libre $\left(\mathrm{NH}_{3}\right)$ situación reportada por Gerardi (2003).

Debido al inicio de la disminución del pH hacia el día 81 e igualmente a la pérdida de alcalinidad y al incremento de $\mathrm{AGV}$, se decidió incorporar al rector un alcalinizante que aportara además, iones de bicarbonato y capacidad tampón (Gerardi, 2003). Se adicionó por tanto la mezcla de $\mathrm{NaHCO}_{3} / \mathrm{CaCO}_{3}$ hasta llevar el pH de 7,2 a 7,6. Aunque esto ocasionó una disminución de AGV desde 16,78 g AcH+/L (día 81) hasta $12,0 \mathrm{~g} \mathrm{AcH}+/ \mathrm{L}$ (día 94) y un leve aumento de la alcalinidad hasta 15,08 gCaCO $/ \mathrm{L}$, el sistema no respondió satisfactoriamente. Por otro lado, la concentración de iones de sodio pudo estar ejerciendo un efecto tóxico (Chen et al., 2008) especialmente sobre las metanogénicas ya que, como se observa en \% (Fig. 3), inmediatamente se adicionó el alcalinizante (día 89), tanto los índices de producción de biogás como los porcentajes de metano en gas cayeron paulatinamente hasta llegar a un valor de $1,2 \quad L_{/} L_{R}$ y $1,7 \%$ respectivamente. Fernández (2008) en su estudio señala que la elevada concentración de AGV puede inhibir la DATS independientemente del $\mathrm{pH}$ haciendo infructuosa la adición de agentes neutralizantes tras una acentuada acidificación.

\section{CONCLUSIONES}

Los resultados obtenidos durante la aclimatación demuestran que el estiércol de vaca empleado como inoculo presenta una elevada actividad metanogénica con un tiempo de aclimatación rápido de cerca de 15 días. Por otro lado, los parámetros de seguimiento durante las etapas posteriores a la de adición de la FORSU revelaron que las proporciones sustrato/inóculo empleada no son capaces de estabilizar el sistema debido a la elevada producción de ácidos grasos volátiles por lo tanto, es necesario explorar estrategias de incremento paulatino de la carga orgánica, de los sólidos totales y/o de los tiempos de retención con el fin de evitar acumulación de metabolitos tóxicos.

\section{AGRADECIMIENTOS}

Al grupo de investigación CYTBIA; al grupo de investigación ASUBAGROIN de la Universidad del Cauca (Colombia) por el apoyo científico y al Sistema General de Regalías (SGR) como ente financiador del proyecto ID-Bioempaques.

\section{REFERENCIAS}

American Public Health Association, American Water Works Association y Water Enviromental Federation, Standard methods for the examination of water and wastewater: selected analytical methods, $20^{\mathrm{a}}$ edición, Baltimore, Estados Unidos (1981)

Ariunbaatar, J.; A. Panico; G. Esposito; F. Pirozzi; y P. Lens, Pretreatment methods to enhance anaerobic digestion of organic solid waste, doi: 10.1016/j.apenergy.2014.02.035, Applied Energy: 123, 143-156, (2014) 
Blanco, D., Tratamiento biológico aerobio-anaerobio-aerobio de residuos ganaderos para la obtención de biogás y compost, Tesis doctoral, Instituto de Medio Ambiente, Recursos Naturales y Biodiversidad, Universidad de León, León-España (2010)

Boontian, N., Optimization of the Anaerobic Digestion of Biomass: A Review, $5^{\text {th }}$ International Conference on Innovations, Recent Trends and Challenges in Mechatronics, Mechanical Engineering and New High-Tech Products Development, 38967-38981, Romania 12 a 13 de septiembre (2013)

Brown, D. and Y. Li, Solid state anaerobic co-digestion of yard waste and food waste for biogas production, doi: 10.1016/j.biortech.2012.09.081, Bioresource Technology: 127, 275-280 (2013)

Canepa, J.R.L. and J.A.S. Olivier, Degradación anaerobia del contenido gástrico ruminal bovino para la obtención de biogás, en un biodigestor tipo cúpula, Ingeniería: 17(1), 57-65, (2013)

Capela, I.; A. Rodrigues; F. Silva; H. Nadais; y L. Arroja, Impact of industrial sludge and cattle manure on anaerobic digestion of the OFMSW under mesophilic conditions, doi: 10.1016/j.biombioe.2007.09.004, Biomass and Bioenergy: 32(3), 245-251 (2008)

Challen-Urbanic, J.M.; B. VanOpstal; and W. Parker, Anaerobic Digestion of the Organic Fraction of Municipal Solid Waste (OFMSW) -Full Scale vs Laboratory Results, doi: 10.5276/JSWTM.2011.33, The Journal of Solid Waste Technology and Management: 37(1), 33-39, (2011)

Chen, Y.; J.J. Cheng; and K.S. Creamer, Inhibition of anaerobic digestion process: A review, doi: 10.1016/j.biortech.2007.01.057, Bioresource Technology: 99(10), 4044-4064 (2008)

Córdoba, V.; M. Fernández; y E. Santalla, The effect of different inoculums on anaerobic digestion of swine wastewater, doi: http://dx.doi.org/10.1016/j.jece.2015.11.003, Journal of Environmental Chemical Engineering: 4(1), 115-122 (2016)

De Baere, L. and B. Mattheeuws, Anaerobic Digestion of the Organic Fraction of Municipal Solid Waste in Europe (2013)

De Francisci, D.; P.G. Kougias; L. Treu; S. Campanaro; and I. Angelidaki, Microbial diversity and dynamicity of biogas reactors due to radical changes of feedstock composition, doi: 10.1016/j.biortech.2014.10.126, Bioresource technology: 176, 56-64 (2015)

El-Fadel, M.; P. Saikaly; and S. Ghanimeh, Startup and Stability of Thermophilic Anaerobic Digestion of OFMSW, Critical Reviews in Environmental Science and Technology: 43(24), 2685-2721 (2013)

Fernández-Güelfo, L.A., Caracterización cinética de la degradación anaerobia termofílica seca de la FORSU: Efecto de diferentes pretratamientos sobre la biodegradabilidad del residuo, Tesis doctoral, Departamento de Ingeniería Química, Tecnología de Alimentos y Tecnologías del Medio Ambiente, Universidad de Cadiz, Cádiz-España (2008)

Forster-Carneiro, T., Digestión Anaerobia Termofílica Seca De Residuos Sólidos Urbanos: Estudio De Las Variables Del Proceso en El Arranque y Estabilización del Bio-Reactor (Tesis doctoral, Departamento de Ingeniería Química, Tecnología de Alimentos y Tecnologías del Medio Ambiente, Universidad de Cadiz, Cádiz-España (2005)

Forster-Carneiro, T.; M. Pérez; L.I. Romero; y D. Sales, Dry-thermophilic anaerobic digestion of organic fraction of the municipal solid waste: Focusing on the inoculum sources, doi: 10.1016/j.biortech.2006.07.008, Bioresource Technology: 98(17), 3195-3203 (2007)

Gerardi, M.H., The Microbiology of Anerobic Digesters, doi: 10.1017/CBO9781107415324.004, 1a edición, Wiley-VCH Verlag GmbH \& Co. KGaA, Nueva Jersey, Estados Unidos (2003)

Jain, S.; S. Jain; I.T. Wolf; J. Lee; and Y.W. Tong, A comprehensive review on operating parameters and different pretreatment methodologies for anaerobic digestion of municipal solid waste, doi: 10.1016/j.rser.2015.07.091, Renewable and Sustainable Energy Reviews: 52, 142-54 (2015)

Khalid, A.; M. Arshad; M. Anjum; T. Mahmood; and L. Dawson, The anaerobic digestion of solid organic waste, doi: 10.1016/j.wasman.2011.03.021, Waste Management: 31(8), 1737-1744 (2011) 
Khanal, S., Anaerobic Biotechnology for Bioenergy Production: Principles and Applications, Wiley-Blackwell, Nueva Delhi, India (2011)

Mao, C.; Y. Feng; X. Wang; and G. Ren, Review on research achievements of biogas from anaerobic digestion, doi: 10.1016/j.rser.2015.02.03, Renewable and Sustainable Energy Reviews: 45, 540-55 (2015)

Motte, J.-C.; R. Escudié; N. Bernet; J.-P. Delgenes; J.-P. Steyer; and C. Dumas, Dynamic effect of total solid content, low substrate/inoculum ratio and particle size on solid-state anaerobic digestion, doi: 10.1016/j.biortech.2013.06.057, Bioresource technology: 144, 141-48 (2013)

Ouda, O.K.M. and S.A. Raza, Waste-to-energy: Solution for Municipal Solid Waste challenges - global perspective, doi: 10.1109/ISTMET.2014.6936517, International Symposium on Technology Management and Emerging Technologies, 270-274, Indonesia 27 al 29 de mayo (2014)

Pavan, P.; P. Battistoni; D. Bolzonella; L. Innocenti; P. Traverso; y F. Cecch, Integration of Wastewater and OFMSW Treatment Cycles: From the Pilot Scale Experiment to the Industrial Realisation - the New Full Scale Plant of Treviso (Italy), Water Science and Technology: 41(12), 165-173 (2000)

Rowse, L.E., Design of small scale anaeroboc digestors for application in rural developing countries, Tesis de Magister en Ciencias, Department of Civil and Environmental Engineering, University of South Florida, FloridaEstados Unidos (2011)

Forster-Carneiro, T.; M. Pérez; and L.I. Romero, Anaerobic digestion of municipal solid wastes: Dry thermophilic performance, doi: 10.1016/j.biortech.2008.03.021, Bioresource Technology: 99(17), 8180-84 (2008)

Valencia, E.; E. Valenzuela; S. González; and C. Vargas, Anaerobic digestion of bovine rumen in laboratory and chemical determination pre and post treatment, Livestock Research for Rural Development: 23(2) (2011)

Yagi, H.; F. Ninomiya; M. Funabashi; and M. Kunioka, Anaerobic Biodegradation of Poly (Lactic Acid) Film in Anaerobic Sludge, doi: 10.1007/s10924-012-0472-z, Journal of Polymers and the Environment: 20(3), 67380 (2012)

Zhu, G.F. and A.K. Jha, Psychrophilic dry anaerobic digestion of cow dung for methane production: Effect of inoculum, doi: 10.2306/scienceasia1513-1874.2013.39.500, Scienceasia: 39(5), 500-510 (2013) 